\title{
Current landscape and future prospects of antiviral drugs derived from microbial products
}

\author{
Naoki Takizawa and Manabu Yamasaki \\ Viral infections are a major global health threat. Over the last 50 years, significant efforts have been devoted to the development \\ of antiviral drugs and great success has been achieved for some viruses. However, other virus infections, such as epidemic \\ influenza, still spread globally and new threats continue to arise from emerging and re-emerging viruses and drug-resistant \\ viruses. In this review, the contributions of microbial products isolated in Institute of Microbial Chemistry for antiviral research \\ are summarized. In addition, the current state of development of antiviral drugs that target influenza virus and hepatitis B virus, \\ and the future prospects for antivirals from natural products are described and discussed.
}

The Journal of Antibiotics (2018) 71, 45-52; doi:10.1038/ja.2017.115; published online 11 October 2017

\section{INTRODUCTION}

Viral infectious diseases cause significant morbidity and mortality in humans. Lower respiratory infections are the deadliest communicable disease, causing 3.2 million deaths globally in 2015. Moreover, in 2015 acquired immunodeficiency syndrome (AIDS) caused by human immunodeficiency virus (HIV) infections killed 1.1 million people and 36.7 million people were infected with HIV globally. To combat viral diseases, both vaccines and antiviral drugs have been employed. As a result of global efforts, smallpox caused by variola virus infections was eradicated in 1979 because of smallpox vaccination (by vaccinia virus). However, the development of effective vaccines is difficult and time consuming for certain viruses that can escape from the host immune response. Therefore, the continual development of antiviral drugs and vaccines is required to combat viral diseases. Approximately 90 drugs have been approved to treat human infectious diseases caused by the following nine viruses: HIV, human cytomegalovirus, hepatitis B virus (HBV), hepatitis C virus (HCV), herpes simplex virus, influenza virus, respiratory syncytial virus, varicella zoster virus and human papillomavirus. In addition, many antiviral drugs are being evaluated in clinical trials. One of the threats of viral infections is emerging and re-emerging viruses, such as Ebola virus, severe acute respiratory syndrome coronavirus, Middle East respiratory syndrome coronavirus and Zika virus. Effective antiviral drugs or vaccines against these emerging viruses are not currently available. Drug-resistant viruses, which can emerge because of the high mutation rate of the viral genome exhibited by many viruses, particularly RNA viruses, also represent a threat to global health.

Almost all currently approved antiviral drugs have been produced by chemical synthesis, but natural products have contributed to the development of antiviral drugs by providing insights into the synthesis of chemical compounds. Viruses must utilize host cellular machineries to propagate and our knowledge of virus-host interactions has recently been advanced. Natural products exhibit great structural diversity and complexity. Thus, both viral and host proteins involved in viral propagation can be targeted to develop antiviral drugs from natural products. In this review, we summarize antiviral microbial products discovered by Institute of Microbial Chemistry (IMC) and discuss antiviral compounds against influenza virus and HBV, because these two viruses threaten global human health now and antiviral drug against these two viruses have been developed. Antiviral compounds isolated in IMC are summarized in Table 1. In addition, we provide a summary of natural compounds for broad-spectrum antivirals and discuss the future potential uses of microbial products as antivirals. Natural compounds with antiviral activities focused in this review are summarized in Table 2.

\section{THE CONTRIBUTIONS OF IMC TO ANTIVIRAL RESEARCH}

Nucleos(t)ide analogs are a major class of approved antiviral drugs that exert therapeutic effects through incorporation into viral DNA and RNA to inhibit virus replication. To date, they have been used as a versatile platform to develop both narrow and broad-spectrum antivirals. Moreover, natural products have provided some motivation to develop new nucleos(t)ide analogs. ${ }^{1,2}$ Two arabinosyl nucleosides, spongouridine and spongothymidine, were isolated from marine sponges and subsequently contributed to the synthesis of vidarabine (arabinosyladenine), which is used for the treatment of herpes simplex virus infections. Professor Umezawa and colleagues identified nucleoside analogs from actinobacteria, such as formycin, ${ }^{3,4}$ coformycin ${ }^{5}$ and oxanosine, ${ }^{6-8}$ some of which exhibit an antiviral activity.

Currently, several classes of anti-HIV drugs are available for HIV treatment and the combination therapies using them, termed highly active antiretroviral therapy that targets multiple steps of the virus life cycle, significantly reduce morbidity and mortality. Zidovudine (azidothymidine) was first approved for HIV treatment by the US 
Table 1 Antiviral compounds isolated in IMC

\begin{tabular}{|c|c|c|}
\hline Compound & Viral infections & Reference \\
\hline Formycins & Influenza virus, poliovirus, vaccinia virus, VSV & 3,4 \\
\hline Coformycin & HIV & 5 \\
\hline Oxanosine & HIV & $6-8$ \\
\hline Benanomicins & HIV & 9 \\
\hline Kijimicin & HIV & 10 \\
\hline Bellenamine & HIV & 11 \\
\hline 14-O-acyladriamycins & $(H I V)^{a}$ & 12 \\
\hline Aminoacridines & $(H I V)^{a}$ & 13 \\
\hline Phleomycin & Poliovirus & 14 \\
\hline Fusaric acid & $\begin{array}{l}\mathrm{HSV}, \mathrm{HBV}, \mathrm{HCV} \text {, varicella-zoster virus, sindbis } \\
\text { virus }\end{array}$ & 15 \\
\hline Aclacinomycin A & Phage $\varphi \times 174$ and $\lambda$ & 16 \\
\hline Clazamycin B & HSV & 17 \\
\hline Sarkomycin & Phage $f 2$ & 18 \\
\hline Leupeptin & 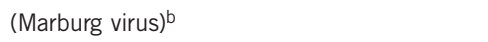 & 21 \\
\hline Antipain & (Poliovirus) $^{\mathrm{c}}$ & 24,25 \\
\hline Elastatinal & ${\text { (Poliovirus })^{c}}$ & 24,25 \\
\hline Pepstatin & $(H I V)^{d}$ & 27,28 \\
\hline Siastatin B & (Influenza virus) $^{\mathrm{e}}$ & 30 \\
\hline
\end{tabular}

Abbreviations: HBV, hepatitis B virus; $\mathrm{HCV}$, hepatitis $\mathrm{C}$ virus; HIV, human immunodeficiency virus; HSV, herpes simplex virus; IMC, Institute of Microbial Chemistry; VSV, vesicular stomatitis virus. alnhibition of HIV reverse transcriptase.

IInhibition of unknown host proteases involved in virus entry.

CInhibition of poliovirus $2 \mathrm{~A}$ protease.

${ }^{d}$ Contributed to the development of anti-HIV drugs against HIV protease.

eInhibition of sialidase activity of influenza virus by epimeric analog of siastatin B.

Table 2 Natural compounds with antiviral activities focused in this review

\begin{tabular}{llc}
\hline Compound & Viral infections & Reference \\
\hline $\begin{array}{l}\text { Spongouridine } \\
\text { Spongothymidine }\end{array}$ & $(\mathrm{HSV})^{\mathrm{a}}$ & 1,2 \\
Statins & $\mathrm{HBV}, \mathrm{HIV}$, influenza virus, DENV, & $101-106$ \\
& $\mathrm{HCMV}, \mathrm{HCV}$ & \\
Myriocin & $\mathrm{HCV}, \mathrm{HBV}$, influenza virus & $108-110$ \\
NA255 & $\mathrm{HCV}$ & 108 \\
Cyclosporine A & HIV, HPV, influenza virus, HBV, HCV, & $81,82,111-$ \\
& coronaviruses, HCMV & 116
\end{tabular}

Abbreviations: DENV, dengue virus; HBV, hepatitis B virus; HCMV, human cytomegalovirus; $\mathrm{HCV}$, hepatitis C virus; HIV, human immunodeficiency virus; HSV, herpes simplex virus; HPV, human papillomavirus.

${ }^{a}$ Contributed to the synthesis of arabinosyladenine.

Food and Drug Administration in 1987. This drug had initially been used for chemotherapeutic intervention against HIV infection, but unmet medical needs of increased efficacy, reduced adverse effects and a higher barrier to drug-resistant mutations remained, prompting a continuing effort to develop new anti-HIV drugs. In this context, IMC had isolated microbial products with anti-HIV activity, such as benanomicins $\mathrm{A}$ and $\mathrm{B},{ }^{9}$ kijimicin, ${ }^{10}$ and bellenamine and its homologs. ${ }^{11}$ Among them, benanomicins also exhibited anti-fungal activity, which may be advantageous for the treatment of AIDS patients with fungal infections. In addition, 14-O-acyladriamycins ${ }^{12}$ and aminoacridines ${ }^{13}$ were synthesized as HIV reverse transcriptase inhibitors. Meanwhile, other research groups found that several compounds discovered by IMC exhibit inhibitory activities against other viruses: phleomycin (anti-poliovirus activity), ${ }^{14}$ fusaric acid (broad-spectrum antiviral activity against herpes simplex virus, varicella-zoster virus, $\mathrm{HBV}, \mathrm{HCV}$ and sindbis virus), ${ }^{15}$ aclacinomycin A (anti-phage $\varphi \mathrm{X} 174$ and $\lambda$ activities), ${ }^{16}$ clazamycin B (anti-herpes simplex virus activity) ${ }^{17}$ and sarkomycin (antiRNA phage f2 activity). ${ }^{18}$ Among them, phleomycins and aclacinomycins were discovered as antibiotics that exhibit antitumor activity, presumably as a consequence of interactions with cellular DNA. ${ }^{19}$

Currently, microbial secondary metabolites are well established to contain many compounds with structural and chemical diversity. Professor Umezawa et al. postulated that certain compounds among them may target enzyme reactions and started to search for enzyme inhibitors from a microbial fermentation broth in 1965. This pioneering work has contributed to various biochemical advances and to therapeutic approaches for certain diseases. For example, in the field of virus research, leupeptin, an inhibitor of serine and cysteine proteases from Streptomyces roseus, ${ }^{20}$ has been shown to prevent glycoprotein-mediated entry of Marburg virus, suggesting the role of unknown host proteases in glycoprotein activation. ${ }^{21}$ Antipain and elastatinal, inhibitors of serine and cysteine proteases from Actinomycetes $^{22,23}$ were used to inhibit poliovirus $2 \mathrm{~A}$ protease. ${ }^{24,25}$ Notably, pepstatin, an aspartic proteinase inhibitor isolated from several species of Streptomyces, ${ }^{26}$ made a significant contribution to the development of a key class of anti-HIV drugs in highly active antiretroviral therapy. HIV proteinase processes viral polyproteins to generate mature proteins and is essential for the virus life cycle. The acetyl-derivative of pepstatin has been reported to be highly effective against HIV protease through a transition state mimic, which led to the rational design of peptide-based protease inhibitors. ${ }^{27,28}$ Furthermore, siastatin $\mathrm{B}$, an analog of sialic acid, was isolated from Streptomyces verticillus var. quantum as a sialidase inhibitor. ${ }^{29}$ Although siastatin B showed no effect on the sialidase activity of influenza virus neuraminidase (NA), its epimeric analog significantly inhibited this enzymatic activity and virus propagation in vitro, as described below. ${ }^{30}$ Together, IMC has identified many microbial products with antiviral activities, some of which have played prominent roles as a unique pharmacophores for antiviral drugs.

\section{INFLUENZA VIRUS}

\section{Influenza virus}

Seasonal influenza is acute respiratory infections caused by influenza viruses. There are three types of seasonal influenza viruses-type A, B and $\mathrm{C}$; influenza A and B viruses cause outbreaks and epidemics. The World Health Organization estimates that annual epidemics caused by influenza $\mathrm{A}(\mathrm{H} 1 \mathrm{~N} 1), \mathrm{A}(\mathrm{H} 3 \mathrm{~N} 2)$ and $\mathrm{B}$ viruses result in $\sim 3$ to 5 million cases of severe illness and 250000 to 500000 deaths, globally. ${ }^{31}$ Furthermore, an influenza pandemic, such as those of the 'Spanish' influenza in 1918 (H1N1), 'Asian' influenza in 1957 (H2N2), 'Hong Kong' influenza in 1968 (H3N2),' 'Russian' influenza in 1977 (H1N1)' and $2009 \mathrm{H} 1 \mathrm{~N} 1$ influenza (swine-origin H1N1), occurs when new influenza A virus against which the human population has no immunity emerges as a consequence of 'genetic reassortment'. ${ }^{32}$ As these epidemics and pandemics still occur, the continued discovery and development of new antiviral drugs and vaccines against influenza infections are required. A total of eight drugs (matrix 2 (M2) inhibitors (amantadine and rimantadine), NA inhibitors (zanamivir, oseltamivir, peramivir and laninamivir octanoate) and viral RNAdependent RNA polymerase inhibitors (ribavirin and favipiravir) have been approved for the treatment of influenza infections. 


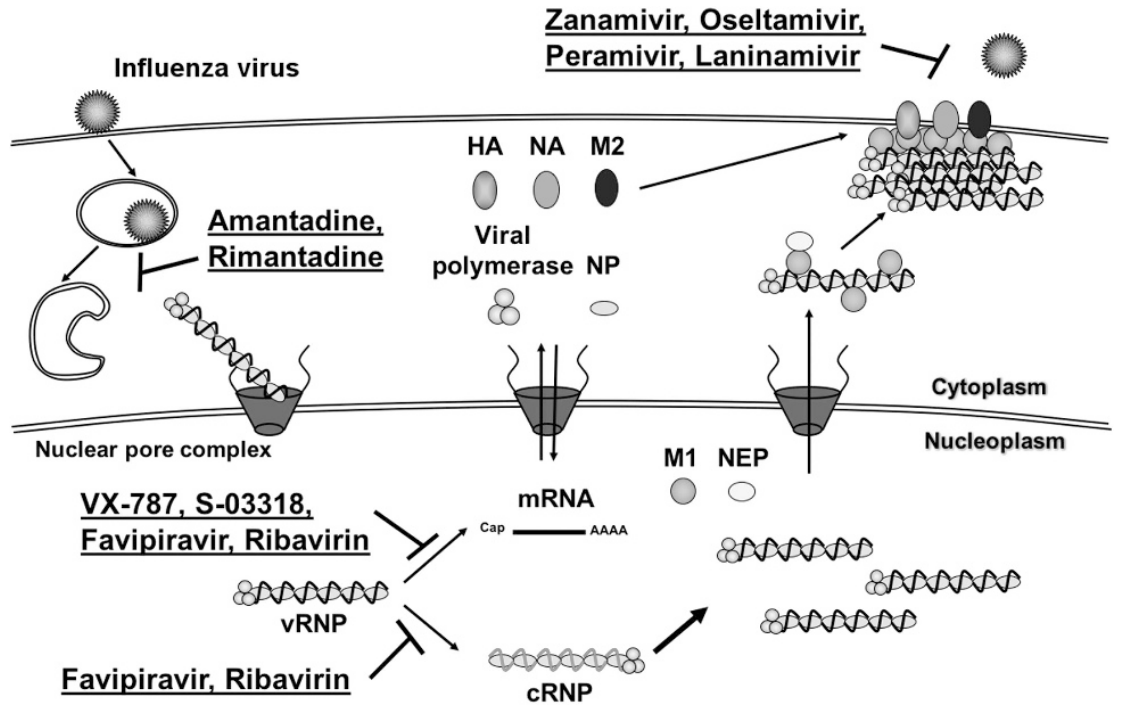

Figure 1 Life cycle of influenza virus and anti-influenza virus drugs. Influenza virus is incorporated into the cell via endocytotic pathway and viral ribonucleoprotein complex (VRNP) which consists of viral genome RNA, viral polymerases and nucleoprotein is released into the cytoplasm. vRNP is transported into the nucleus where transcription and replication occur. In replication step, complementary RNA is synthesized, and then, progeny VRNPs are synthesized from complementary RNP (cRNP). Progeny vRNPs are transported to cytoplasm by binding to matrix protein 1 (M1) and nuclear export protein (NEP). Budding of progeny virion is occurred under plasma membrane with viral membrane proteins (HA, NA and M2). Amantadine and rimantadine inhibit M2 ion channel activity, and therefore, inhibit release of vRNP into cytoplasm. VX-787 and S-033188 inhibit transcription of viral mRNA by binding to viral polymerase complex. Favipiravir and ribavirin inhibit viral RNA synthesis. Zanamivir, oseltamivir, peramivir and laninamivir octanoate all inhibit neuraminidase activity of NA by binding to the active center of the enzyme, and therefore, inhibit release of progeny virion from the cell surface. A Full color version of this figure is available at Journal of Antibiotics online.

\section{M2 inhibitors}

1-Adamantanamine (amantadine) was the first synthetic compound shown to inhibit influenza virus propagation ${ }^{33}$ and was approved in 1966 to treat influenza A virus infections. The target of amantadine is M2 integral membrane protein, which exhibits $\mathrm{H}^{+}$ion channel activity. Acidification of virus-containing endosomes activates M2 ion channel activity. ${ }^{34}$ Acidification in the virion interior is necessary to allow the viral genome to diffuse away from the endosomal membrane. Amantadine inhibits proton translocation by binding to the transmembrane domain of the M2 protein tetramer, thereby inducing the release of the viral genome into the cytoplasm ${ }^{35}$ (Figure 1). However, amantadine and rimantadine, a derivative of amantadine, are not currently used to treat influenza virus infections because drug-resistant viruses can emerge rapidly (both the $\mathrm{A} / \mathrm{H} 1 \mathrm{~N} 1 p d m$ and $\mathrm{A} / \mathrm{H} 3 \mathrm{~N} 2$ seasonal epidemic strains have the amantadine-resistance S31N mutation) and the central nervous system side effects are particularly common in patients treated with amantadine.

\section{NA inhibitors}

Hemagglutinin (HA) and NA are two major glycoproteins expressed by both influenza A and B viruses. HA mediates the binding of viruses to target cells via terminal sialic acid and NA cleaves terminal sialic acids from host cells. NA activity facilitates the release of progeny influenza virus by digesting sialic acids in the HA receptors (Figure 1). The sialidase activity of NA has been targeted in the development of anti-influenza drugs. Siastatin B is an analog of sialic acid and that inhibits the sialidase activity of Clostridium perfrigens NAs, but does not inhibit that of influenza virus (A/Aichi/2/68 strain) NA and Newcastle disease virus (Sato strain) $\mathrm{HN}^{29}$ A siastatin B epimeric analog, 3-episiastatin B, can inhibit the sialidase activity of influenza A and $\mathrm{B}$ viruses NA and propagation of influenza A virus. ${ }^{30}$
A synthetic analog of neuraminic acid, 2,3-didehydro-2-deoxy- $N$ acetylneuraminic acid (Neu5Ac2en), was found to be an inhibitor of influenza virus NA. ${ }^{36}$ Neu5Ac2en is considered to be a transition state analog that can bind to the active site of NA. ${ }^{37-39}$ Based on a structural analysis of the NA-Neu5Ac2en complex, analogs of Neu5Ac2en were synthesized and the specificity for influenza virus NA and activity of these analogs were tested. Among these analogs, 4-guanidinoNeu5Ac2en (zanamivir) exhibited antiviral activity against various influenza A and B viruses. ${ }^{40}$ The guanidine group in zanamivir is suggested to form salt bridges with Glu119 in the NA active site and strong charge-charge interactions with Glu227 of NA. Because of poor oral bioavailability, zanamivir is administered by inhalation. To improve oral bioavailability, novel analogs were synthesized based on X-ray crystallographic analysis of NA with key analogs. ${ }^{41}$ One analog, GS4071, inhibited influenza A virus NA activity. The NA inhibition activity of GS4071 was comparable to that of zanamivir. Oseltamivir is an ethylester prodrug of GS4071. The oral bioavailability of oseltamivir was improved compared with that of GS4071. ${ }^{42}$ After zanamivir and oseltamivir, two NA inhibitors, peramivir and laninamivir octanoate, were launched. ${ }^{43,44}$ Peramivir is administered by intravenous administration and laninamivir octanoate is administered by inhalation. These two drugs are used as a single-dose treatment for influenza $\mathrm{A}$ and $\mathrm{B}$ viruses.

\section{Virus polymerase inhibitors}

Viral polymerase complex is a promising target for the development of anti-influenza drugs, because transcription and replication are critical steps for virus propagation. Moreover, the viral polymerase complex acts by a specific molecular mechanism for viral transcription and replication. The viral polymerase complex is a heterotrimer composed of three subunits: PA, PB1 and PB2. At the initiation of viral mRNA transcription, influenza viral polymerase cannot synthesize the $5^{\prime}$-cap structure of mRNA that is necessary for translation and uses a unique 
'cap-snatching' mechanism to 'steal' the cap structure of mRNA from host mRNAs. ${ }^{45}$ Host-cell pre-mRNA is bound to the PB2 subunit via its $5^{\prime}$-cap structure and can be cleaved at nucleotide $10-13$ by the endonuclease activity of the PA subunit. Using this capped fragment as a primer, the viral polymerase complex synthesizes viral mRNA. These processes have been targeted in the development of anti-influenza drugs and two inhibitors, VX-787 (pimodivir) and S-033188, are under phase 2 and 3 clinical trials, respectively. VX-787 is a PB2 inhibitor that binds to the cap-binding pocket in PB2. ${ }^{46,47}$ The molecule 3-pyrimidineazainodole, VRT-0761704, was identified as a lead compound for anti-influenza virus activities by phenotypic screening, and the target of this compound was determined to be the PB2 subunit. Based on structural information for the PB2- $\mathrm{m}^{7} \mathrm{GTP}$ and PB2-VRT-0761704 complex, VX-787 was synthesized. VX-787 represents an orally bioavailable compound that is only effective for influenza A virus, not influenza B virus. S-03318 specifically inhibits the endonuclease activity of PA and thus prevents the cleavage of host pre-mRNAs. S-03318 is effective for both influenza A and influenza B virus infections.

Two approved drugs, ribavirin and T-705 (favipiravir), inhibit influenza viral RNA synthesis (Figure 1). Ribavirin is a synthetic nucleoside analog that is metabolized to the triphosphate form (Ribavirin-TP). ${ }^{48}$ Ribavirin-TP inhibits the RNA polymerase complex of influenza virus by competing with ATP and GTP. ${ }^{49}$ Ribavirin has not been used to treat influenza virus infection, but has been used for HCV infections along with pegylated interferon. T-705 is a pyrazine derivative that is metabolized to T705-4-ribofuranosyl-5'-triphosphate in cells. ${ }^{50,51}$ T705-4-ribofuranosyl- $5^{\prime}$-triphosphate acts as a specific inhibitor of influenza virus RNA synthesis. T705-4-ribofuranosyl-5'triphosphate directly inhibits viral RNA synthesis and is incorporated into synthesized RNA in the monophosphate form (T-705RMP) without chain termination. ${ }^{52-54}$ The incorporated T-705RMP can induce the mis-incorporation of incorrect nucleotides, so a large fraction of progeny virions from T-705-treated cells do not exhibit infectivity. T-705 has been approved for use against pandemic influenza infections in Japan.

\section{Natural products for anti-influenza virus infections}

All of the approved and clinical trial drugs mentioned above are synthesized, but natural products for anti-influenza virus drugs have been screened and studied. NA has been targeted for the anti-influenza virus activity of natural products because high-throughput assay systems for measurements of NA activity have been constructed. Reported natural products that show inhibition of NA can be classified into five groups: flavonoids, oligostibenes, coumarins, diarylheptanoids and others. ${ }^{55} \mathrm{HA}$ is also targeted for the screening of antiinfluenza virus drugs. A novel compound with a pentacyclic structure, stachyflin isolated from Stachybotrys, inhibits conformational changes of $\mathrm{HA}$ at low $\mathrm{pH}$, thereby inhibiting membrane fusion between virus and host cells. ${ }^{56-58}$

\section{HEPATITIS B VIRUS}

According to the World Health Organization estimates in 2016, the annual number of deaths resulting from viral hepatitis is $\sim 1.4$ million, which is comparable to that of HIV and tuberculosis; $47 \%$ of those deaths can be attributed to HBV. ${ }^{59}$ Approximately 257 million individuals are chronically infected with HBV worldwide and have an elevated risk of developing liver cirrhosis and hepatocellular carcinoma. ${ }^{60}$ Current antiviral drugs approved by the US Food and Drug Administration include immunomodulators (interferon- $\alpha$ and pegylated interferon- $\alpha$ ) and nucleos $(\mathrm{t}$ )ide analogs (lamivudine, adefovir, telbivudine, entecavir and tenofovir), which are useful for therapeutic treatment to interrupt the disease progression. However, these drug classes have certain limitations; for immunomodulators, a partial efficacy and side effects exist, and for nucleos $(t)$ ide analogs, the emergence of drug-resistant virus strains can occur during long-term use. Thus, an unmet need remains for the development of novel antiHBV drugs. Although microbial products with anti-HBV activities, as compared with phytochemicals, ${ }^{61,62}$ are limited to date, microbial metabolites may represent a fundamental source for compounds that interfere with the virus life cycle, because of structural diversity and various biological activities.

\section{HBV life cycle}

HBV is a small enveloped hepatotropic DNA virus with an $\sim 3.2 \mathrm{~kb}$ partially double-stranded, relaxed circular DNA genome that encodes polymerase, precore (HBV e antigen), core (capsid), surface antigens (small, middle and large) and HBx protein (transcriptional activator). As shown in Figure 2, the virus enters hepatocytes by binding to the cellular receptor(s), which includes sodium taurocholate cotransporting polypeptide. ${ }^{63}$ Following release of the nucleocapsid, relaxed circular DNA is transported into the nucleus and converted into covalently closed circular DNA (cccDNA). The cccDNA is quite stable in the form of a minichromosome and serves as a template for the transcription of all viral mRNAs. Among these mRNAs, the $3.5 \mathrm{~kb}$ pregenomic RNA is translated into polymerase and core proteins, which is then encapsidated together with polymerase that serves as a RNA replicative intermediate. The pregenomic RNA is reverse transcribed into minus-strand DNA and degraded by the RNase $\mathrm{H}$ activity associated with the polymerase during this process. HBV polymerase subsequently synthesizes plus-strand DNA with various lengths, which generates relaxed circular DNA. Finally, the matured nucleocapsids are released from infected cells after the acquisition of an envelope that contains the surface antigens in the endoplasmic reticulum and Golgi apparatus to produce progeny virions, or to be recycled to the nucleus to supply the cccDNA pool.

To identify promising lead compounds for the drug development, it is important to select potential therapeutic targets for chronic HBV infection from the virus life cycle. Although various anti-HBV drugs, which is beyond the scope of this review, are the subject of preclinical and clinical trials, ${ }^{64,65}$ their inhibitory mechanisms should provide helpful data to make reasonable selections of such key targets for the drug screening for microbial products.

\section{Potential applications of microbial products in anti-HBV drug development}

Approved nucleos(t)ide analogs competitively inhibit DNA elongation of viral polymerase after the conversion to triphosphate form by cellular enzymes. Although nucleos(t)ide analog inhibitors can be highly effective in preventing liver disease progression, these drugs are not curative and require long-term use, which is associated with a risk of drug-resistance and side-effect. To overcome these drawbacks, several nucleos $(\mathrm{t})$ ide analogs are currently in preclinical and clinical trials, which indicates that active site of the DNA polymerization remains an indispensable drug target for $\mathrm{HBV}$ treatment. Indeed, a new tenofovir derivative, tenofovir alafenamide (Vemlidy), was recently approved and its improved pharmacological properties contribute to the reduction of nephrotoxicity. To date, microbial metabolites have yielded various nucleos(t)ide analogs, as mentioned above, including oxetanocin $\mathrm{A}$ and its derivative that exhibits antiHBV activity. ${ }^{66,67}$ 


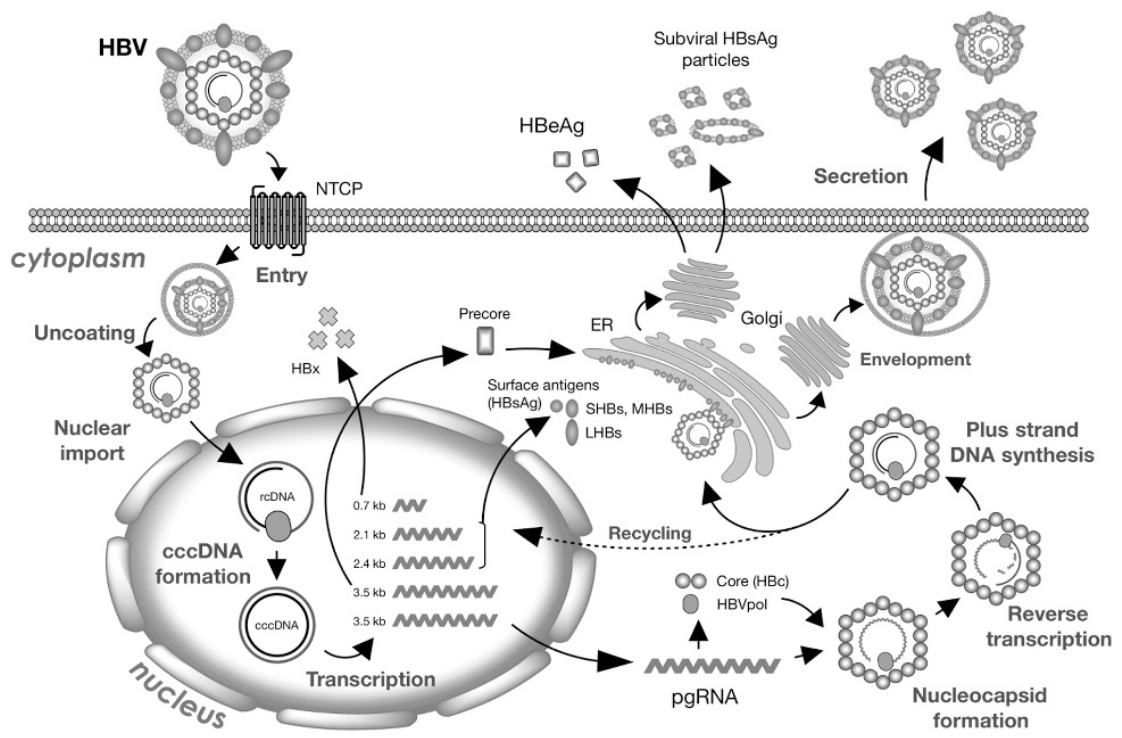

Figure 2 Life cycle of hepatitis B virus (HBV). HBV enters hepatocytes following binding to the cellular receptor(s) including sodium taurocholate cotransporting polypeptide (NTCP). After uncoating, relaxed circular DNA (rcDNA) within a released nucleocapsid is transported into the nucleus due to nuclear localization signal on the capsid. In the nucleus, rcDNA is converted into cccDNA by viral and cellular enzymes. The cccDNA is quite stable in the form of a minichromosome that is responsible for persistent HBV infection and transcribed by host RNA polymerase II to generate four mRNAs as follows: $3.5 \mathrm{~kb}$ pregenomic RNA (pgRNA) and precore mRNA, 2.4 and $2.1 \mathrm{~kb}$ surface antigens mRNA, and $0.7 \mathrm{~kb} \mathrm{HBx}$ mRNA. pgRNA is translated into the viral polymerase and core proteins, which is then encapsidated together with polymerase. Within the nucleocapsid, the pgRNA is reverse transcribed into minusstrand DNA and degraded by the RNase $\mathrm{H}$ activity associated with the polymerase followed by plus-strand DNA synthesis. The 2.4 and $2.1 \mathrm{~kb}$ mRNA are translated into large (LHBs), and middle (MHBs) and small (SHBs) surface antigens, respectively. Finally, the matured nucleocapsids are enveloped by lipid bilayer containing these surface antigens at the endoplasmic reticulum (ER) and Golgi apparatus and progeny virions are released from infected cells. In parallel, a huge amounts of subviral HBsAg particles are assembled in the absence of nucleocapsid and released. The other $3.5 \mathrm{~kb}$ mRNA is translated into precore protein that is proteolytically processed to $\mathrm{HBeAg}$. $\mathrm{HBx}$ has a multifunctional role in activation of various viral and cellular promoters and enhancers. A Full color version of this figure is available at Journal of Antibiotics online.

The ideal goal of anti-HBV treatments is the loss of viral surface antigens, HBsAg, which is correlated with dramatic reduction of incidence of hepatocellular carcinoma. However, current nucleos $(\mathrm{t})$ ide inhibitors are ineffective in this regard, because they have little effects on the cccDNA pool that serves as the template for HBsAg expression. To improve treatment outcomes, novel anti-HBV agents that target other steps of viral DNA synthesis, in combination with current HBV drugs, may promote cccDNA decay and provide a higher barrier to resistance. ${ }^{65,68}$ The core protein self-assembles to form a nucleocapsid in which viral DNA synthesis is carried out, and several inhibitors of this process are in phase I/II clinical trials. Furthermore, such inhibitors may facilitate cccDNA decay because the strong inhibition of viral DNA synthesis is thought to completely block the recycling of nucleocapsids into the nucleus for cccDNA replenishment. ${ }^{69,70}$ Thus, perturbing the capsid assembly process would be a promising approach for novel HBV treatment. A derivative of leucamide A, a cyclic heptapeptide derived from sponge, was found to interfere with this process. ${ }^{71,72}$ Furthermore, RNase H-mediated pregenomic RNA degradation is another essential step for the viral DNA synthesis and a novel inhibitor of this activity is undergoing preclinical trials. ${ }^{64}$ As the nucleolytic cleavage is dependent on a metal ion coordinated by the active site of RNase $\mathrm{H}$, a class of metal-chelating inhibitors might have a potential for the development of novel RNase $\mathrm{H}$ inhibitors. Although $\beta$-thujaplicinol, which showed inhibitory activity toward HBV RNase $\mathrm{H}$, is of plant origin, ${ }^{73,74}$ such hydroxylated tropolones are often found in microbial metabolites. ${ }^{75}$ Taken together, it appears likely that microbial metabolites represent an enthralling resource of new chemical entities that target HBV DNA synthesis. In general, natural product screening is a time-consuming process that requires the fractionation and isolation of active compound(s) from complex extracts of an unknown composition. Recently, we developed a system to facilitate the detection of the replicating HBV genome using adenovirus vectors, ${ }^{76}$ which can be used to quantitatively evaluate the inhibitory potency of a test compound in a shorter period of time than other model systems for studying HBV replication using cell lines. Similarly, new efficient detection methods for virus replication would allow for the rapid drug screening of natural resources.

As HBV infection is significantly influenced by various of host factors, host factor-targeted antivirals are prospective therapeutic approaches. ${ }^{77,78}$ Myrcludex-B, a peptide that mimics the N-terminus of the preS1 in the large surface antigen that is involved in binding to sodium taurocholate cotransporting polypeptide, can block virus entry to hepatocytes and is currently in phase II clinical trials. ${ }^{79,80}$ Recently, cyclosporine A, a fungal cyclic polypeptide, and its analogs have been reported to inhibit the virus entry by targeting sodium taurocholate cotransporting polypeptide. ${ }^{81,82}$ Such macrocyclic peptide scaffolds often have the advantage of occupying an interface that is required for protein-protein interactions, such as receptor binding and capsid assembly, as described above. Moreover, host enzymes that are essential for virus replication represent promising drug targets. Deoxynojirimycin is an iminosugar $\alpha$-glucosidase inhibitor and its analogs have shown to reduce the HBV secretion from infected cells. ${ }^{83,84}$ Iminosugars have a potential for development as antiviral drugs that disrupt the folding of viral glycoproteins by inhibiting host glucosidase, which is localized to the endoplasmic reticulum..$^{85}$ Deoxynojirimycin is of plant origin, but this class of compounds is also found in microbial metabolites, such as siastatin B as described above. ${ }^{29}$ Although the effects of siastatin B on HBV replication are 
presently unknown, host glucosidase involved in the antigen and virion secretion might be a suitable target for anti-HBV drug screening for microbial metabolites. Furthermore, several therapeutic vaccines for the treatment of chronic HBV infection are in preclinical and clinical trials. This approach aims to stimulate either specific or nonspecific immune responses against $\mathrm{HBV}$ to eliminate the cccDNA pool, while adjuvants may work to enhance vaccination. Previously, bestatin (Ubenimex), an immunomodulator discovered by IMC, 86 has been reported to enhance immune responses for HIV vaccination in a murine model, ${ }^{87}$ although its effect on HBV vaccination is unknown. Compounds with adjuvant activity on HBV vaccination might be present in microbial metabolites.

\section{FUTURE PROSPECTS FOR NATURAL PRODUCTS IN ANTIVIRAL DRUG DEVELOPMENT}

Recently, deadly disease outbreaks caused by emerging and re-emerging viruses have become increasingly threatening, including outbreaks of Nipah virus in Malaysia, Hendra virus in Australia, Hantavirus in the United States, Ebola virus in Africa, severe acute respiratory syndrome and Middle East respiratory syndrome coronaviruses in China and Mideast, Zika virus in Oceania and Americas, and the influenza virus H5N1, H1N1 and H7N2 subtypes. No efficient vaccine or specific antiviral drug for these emerging and re-emerging virus diseases is yet available. Not only antiviral drugs against specific virus infections, but also broad-spectrum antiviral drugs need to be developed. Here we summarize candidates of broad-spectrum antiviral drugs, nucleoside analogs and drugs against host proteins and discuss the potential of natural compounds for broad-spectrum antiviral drugs.

Nucleoside analogs are candidates for use as broad-spectrum antiviral drugs. Ribavirin is known to be a broad-spectrum RNA virus inhibitor and an antiviral candidate for emerging infectious diseases, such as dengue virus, norovirus, and Hendra and Nipah viruses. ${ }^{88-90}$ The potential activity of ribavirin against broad-spectrum RNA viruses can be exerted by both the direct and indirect inhibition of viral polymerase activity. Ribavirin monophosphate inhibits inosine- $5^{\prime}$-monophosphate dehydrogenase, which catalyzes the reaction from inosine- $5^{\prime}$-monophosphate to xanthosine monophosphate, thereby decreasing intracellular GTP levels. ${ }^{91}$ Nucleotide pool imbalances that result from ribavirin treatment could cause a reduction of viral polymerase activity and the incorporation of alternative nucleotides for GTP. Recent reports suggest that T-705 is also a broadspectrum RNA virus inhibitor for viruses such as Ebola virus. ${ }^{92,93}$ The detailed mechanism of the anti-viral activity of T-705 against these RNA viruses is not known and merits further study. Both BCX4430 and GS-5734 are nucleoside analogs and are the subject of clinical trials for Ebola virus disease. ${ }^{94,95}$ They also exhibit broad-spectrum antiviral effects against RNA virus families, including filoviruses, arenaviruses, paramyxoviruses and flaviviruses. ${ }^{96-99}$ As mentioned above, natural products have contributed to the development of antiviral nucleotide analogs. New classes of nucleotide analogs derived from natural products will be promising lead compounds for emerging viruses.

Drugs against non-viral proteins (that is, host proteins) are also broad-spectrum antiviral drug candidates. Viruses use common cellular machinery from entry into to exit from host cells. Inhibitors against such cellular machinery could inhibit viral propagation. These host targeted drugs are not yet approved as antivirals, but research and development are ongoing. RNA viruses are known to use host membrane compartments for replication and trafficking. Thus, lipid metabolism is a candidate target of antiviral drugs. Statins, which were first isolated from Penicillium citrinum, are hydroxymethylglutaryl- coenzyme A reductase inhibitors. Treatment with statins decreases levels of low-density lipoprotein cholesterol. ${ }^{100}$ Antiviral effects of stains have been reported for HBV, HIV, influenza virus, dengue virus, human cytomegalovirus and HCV. ${ }^{101-106}$ The antiviral activity of statins may be a consequence of disruption of the membrane components that viruses use, along with changes in membrane trafficking. Serine palmitoyltransferase inhibitors, myriocin isolated from Myriococcum albomyces ${ }^{107}$ and NA255 isolated from Fusarium incarnatum, ${ }^{108}$ can also inhibit the propagation of HCV, HBV and influenza virus. ${ }^{108-110}$ Serine palmitoyltransferase acts in the sphingomyelin synthesis pathway and sphingomyelin is an essential component of 'lipid rafts'. Myriocin inhibits the intracellular transport of influenza virus glycoproteins and NA255 disrupts the HCV replication complex.

Drug repositioning (also known as drug repurposing) is of growing interest to reduced time and cost associated with drug developments. Cyclosporine A (CsA) is an approved immunosuppressive drug and it has been revealed that CsA is effective against viruses such as HIV, human papillomavirus, influenza virus, HCV, coronaviruses and human cytomegalovirus. ${ }^{111-116} \mathrm{CsA}$ can be isolated from Hypocladium inflatum gams and inhibits prolyl isomerase cyclophilins, which are implicated in the regulation of protein conformation. ${ }^{117}$ The antiviral mechanism of CsA involves the inhibition of the correct folding of viral proteins by cyclophilins. CsA exerts immunosuppressive effects as the CsA-cyclophilin A complex is formed by the inhibition of calcium-dependent phosphatase activity by calcineurin, which leads to the inhibition of key activators of T cells. Therefore, CsA derivatives that lack the inhibitory activity of calcineurin, but maintain the inhibitory activity of cyclophilins have been semi-synthesized. ${ }^{118} \mathrm{~A}$ CsA derivative, alisporivir (DEB025), with ribavirin has been studied as an anti-HCV drug and is the subject of a clinical trial. ${ }^{119}$

Today, viral proteins are the major target of antiviral drugs. Antiviral drugs against specific target proteins have been successful against important infectious diseases, such as HIV and HCV. With rapidly increasing knowledge of protein structure, development of in silico tools, and methods for chemical and genetic modification, more antiviral drugs will be developed that will contribute to the future elimination of specific infectious diseases. Concurrently, we now face the threat of emerging and re-emerging infectious diseases with no available specific antiviral drug. To prevent a pandemic of these infections, broadspectrum antiviral drugs also need to be developed. Natural products are a rich source and promising starting points to identify lead compounds for antivirals that target host proteins. Studies of virushost interactions and 'omics' data from virus infected cells will help us to accelerate the development of screening systems and the identification of potential drug targets. We anticipate that novel antiviral drugs derived from microbial products will contribute to both the elimination of specific virus infections and emerging virus infections in the future.

\section{DEDICATION}

We dedicate this review to Prof Hamao Umezawa.

\section{CONFLICT OF INTEREST}

The authors declare no conflict of interest.

\section{ACKNOWLEDGEMENTS}

We thank all members and alumni of IMC, particularly Dr Akio Nomoto.

1 De Clercq, E. \& Li, G. Approved antiviral drugs over the past 50 years. Clin. Microbiol. Rev. 29, 695-747 (2016). 
2 Gogineni, V., Schinazi, R. F. \& Hamann, M. T. Role of marine natural products in the genesis of antiviral agents. Chem. Rev. 115, 9655-9706 (2015).

3 Hori, M., Takita, T., Koyama, G., Takeuchi, T. \& Umezawa, H. A new antibiotic, Formycin. J. Antibiot. (Tokyo) 17, 96-99 (1964).

4 Takeuchi, T., Iwanaga, J., Aoyagi, T. \& Umezawa, H. Antiviral effect of formycin and formycin B. J. Antibiot. (Tokyo) 19, 286-287 (1966).

5 Sawa, T., Fukagawa, Y., Homma, I., Takeuchi, T. \& Umezawa, H. Mode of inhibition of coformycin on adenosine deaminase. J. Antibiot. (Tokyo) 20, 227-231 (1967).

6 Shimada, N. et al. Oxanosine, a novel nucleoside from actinomycetes. J. Antibiot. (Tokyo) 34, 1216-1218 (1981).

7 Saito, Y. et al. Synthesis and anti-HIV activity of unusual nucleoside oxanosine derivatives. Nucleic Acids Symp. Ser. 19-20 (1999) at http://www.ncbi.nlm.nih. gov/pubmed/10780358.

8 Nakamura, H. et al. The X-ray structure determination of oxanosine. J. Antibiot. (Tokyo) 34, 1219-1221 (1981).

9 Hoshino, H., Seki, J. \& Takeuchi, T. New antifungal antibiotics, benanomicins A and $B$ inhibit infection of T-cell with human immunodeficiency virus (HIV) and syncytium formation by HIV. J. Antibiot. (Tokyo) 42, 344-346 (1989).

10 Nakamura, M., Ohno, T., Kunimoto, S., Naganawa, H. \& Takeuchi, T. Kijimicin: an inhibitor of human immunodeficiency virus in acutely and chronically infected cells. J. Antibiot. (Tokyo) 44, 569-571 (1991).

11 Kondo, S. et al. New bellenamine homologs inhibiting human immunodeficiency virus type I infectivity. J. Antibiot. (Tokyo) 49, 113-118 (1996).

12 Ajito, K. et al. Inhibition of human immunodeficiency virus-associated reverse transcriptase by 14-0-acyladriamycins. J. Antibiot. (Tokyo) 42, 611-619 (1989).

13 Atsumi, S. et al. Inhibition of human immunodeficiency virus-associated reverse transcriptase by aminoacridines. Drugs Exp. Clin. Res. 14, 719-722 (1988).

14 Koch, G. Differential effect of phleomycin on the infectivity of poliovirus and poliovirus-induced ribonucleic acids. J. Virol. 8, 28-34 (1971).

15 Fernandez-Pol, J. A. Conservation of multifunctional ribosomal protein metallopanstimulin-1 (RPS27) through complex evolution demonstrates its key role in growth regulation in Archaea, eukaryotic cells, DNA repair, translation and viral replication. Cancer Genomics Proteomics 8, 105-126 (2011).

16 Tanaka, A., Sen, K., Morita, J. \& Komano, T. Phage inactivation by aclacinomycin A and its analogues. J. Antibiot. (Tokyo) 36, 1242-1244 (1983).

17 Dolak, L. A. \& DeBoer, C. Clazamycin B is antibiotic 354. J. Antibiot. (Tokyo) 33, 83-84 (1980).

18 Koenuma, M., Kinashi, H. \& Otake, N. An improved screening method for antiphage antibiotics and isolation of sarkomycin and its relatives. J. Antibiot. (Tokyo) 27 801-804 (1974).

19 Kunimoto, T., Hori, M. \& Umezawa, H. Modes of action of phleomycin, bleomycin and formycin on HeLa S3 cells in synchronized culture. J. Antibiot. (Tokyo) 20 277-281 (1967).

20 Aoyagi, T., Takeuchi, T., Matsuzaki, A., Kawamura, K. \& Kondo, S. Leupeptins, new protease inhibitors from Actinomycetes. J. Antibiot. (Tokyo) 22, 283-286 (1969).

21 Gnirß, K. et al. Cathepsins B and L activate Ebola but not Marburg virus glycoproteins for efficient entry into cell lines and macrophages independent of TMPRSS2 expression. Virology 424, 3-10 (2012).

22 Suda, H., Aoyagi, T., Hamada, M., Takeuchi, T. \& Umezawa, H. Antipain, a new protease inhibitor isolated from actinomycetes. J. Antibiot. (Tokyo) 25, 263-266 (1972).

23 Umezawa, H., Aoyagi, T., Okura, A., Morishima, H. \& Takeuchi, T. Elastatinal, a new elastase inhibitor produced by actinomycetes. J. Antibiot. (Tokyo) 26 787-789 (1973).

24 Molla, A., Hellen, C. U. \& Wimmer, E. Inhibition of proteolytic activity of poliovirus and rhinovirus $2 \mathrm{~A}$ proteinases by elastase-specific inhibitors. J. Virol. 67, 4688-4695 (1993).

25 Belov, G. A. et al. Bidirectional increase in permeability of nuclear envelope upon poliovirus infection and accompanying alterations of nuclear pores. J. Virol. 78 10166-10177 (2004).

26 Umezawa, H., Aoyagi, T., Morishima, H., Matsuzaki, M. \& Hamada, M. Pepstatin, a new pepsin inhibitor produced by Actinomycetes. J. Antibiot. (Tokyo) 23, 259-262 (1970)

27 Richards, A. D., Roberts, R., Dunn, B. M., Graves, M. C. \& Kay, J. Effective blocking of HIV-1 proteinase activity by characteristic inhibitors of aspartic proteinases. FEBS Lett. 247, 113-117 (1989).

28 Roberts, N. A. et al. Rational design of peptide-based HIV proteinase inhibitors. Science 248, 358-361 (1990).

29 Umezawa, H., Aoyagi, T., Komiyama, T., Morishima, H. \& Hamada, M. Purification and characterization of a sialidase inhibitor, siastatin, produced by Streptomyces. J. Antibiot. (Tokyo) 27, 963-969 (1974).

30 Nishimura, Y. et al. Synthesis of 3-episiastatin B analogues having anti-influenza virus activity. J. Antibiot. (Tokyo) 46, 1883-1889 (1993).

31 World Health Organization Media centre. Influenza (Seasonal) Fact sheet. http://www. who.int/mediacentre/factsheets/fs211/en/.

32 Lowen, A. C. Constraints, drivers, and implications of influenza a virus reassortment. Annu. Rev. Virol. (e-pub ahead of print; doi: 10.1146/annurev-virology-101416041726) (2017)

33 Davies, W. L. et al. Antiviral activity of 1 -adamantanamine (amantadine). Science 144, 862-863 (1964).

34 Liang, R., Li, H., Swanson, J. M. J. \& Voth, G. A. Multiscale simulation reveals a multifaceted mechanism of proton permeation through the influenza A M2 proton channel. Proc. Natl Acad. Sci. USA 111, 9396-9401 (2014).
35 Hong, M. \& Degrado, W. F. Structural basis for proton conduction and inhibition by the influenza M2 protein. Nature 451, 596-599 (2008).

36 Meindl, P., Bodo, G., Palese, P., Schulman, J. \& Tuppy, H. Inhibition of neuraminidase activity by derivatives of 2-deoxy-2,3-dehydro- $\mathrm{N}$-acetylneuraminic acid. Virology 58, 457-463 (1974).

37 Janakiraman, M. N., White, C. L., Laver, W. G., Air, G. M. \& Luo, M. Structure of influenza virus neuraminidase $\mathrm{B} / \mathrm{Lee} / 40$ complexed with sialic acid and a dehydro analog at 1.8-A resolution: implications for the catalytic mechanism. Biochemistry 33 , 8172-8179 (1994)

38 Taylor, N. R. \& von Itzstein, M. Molecular modeling studies on ligand binding to sialidase from influenza virus and the mechanism of catalysis. J. Med. Chem. 37, 616-624 (1994).

39 Chong, A. K., Pegg, M. S. \& von Itzstein, M. Characterisation of an ionisable group involved in binding and catalysis by sialidase from influenza virus. Biochem. Int. 24, 165-171 (1991)

40 von Itzstein, M. et al. Rational design of potent sialidase-based inhibitors of influenza virus replication. Nature 363, 418-423 (1993).

$41 \mathrm{Kim}, \mathrm{C}$. U. et al. Influenza neuraminidase inhibitors possessing a novel hydrophobic interaction in the enzyme active site: design, synthesis, and structural analysis of carbocyclic sialic acid analogues with potent anti-influenza activity. J. Am. Chem. Soc. 119, 681-690 (1997).

42 Eisenberg, E. J., Bidgood, A. \& Cundy, K. C. Penetration of GS4071, a novel influenza neuraminidase inhibitor, into rat bronchoalveolar lining fluid following oral administration of the prodrug GS4104. Antimicrob. Agents Chemother. 41, 1949-1952 (1997).

43 Smee, D. F., Huffman, J. H., Morrison, A. C., Barnard, D. L. \& Sidwell, R. W. Cyclopentane neuraminidase inhibitors with potent in vitro anti-influenza virus activities. Antimicrob. Agents Chemother. 45, 743-748 (2001).

44 Yamashita, M. et al. CS-8958, a prodrug of the new neuraminidase inhibitor R-125489, shows long-acting anti-influenza virus activity. Antimicrob. Agents Chemother. 53, 186-192 (2009).

45 te Velthuis, A. J. W. \& Fodor, E. Influenza virus RNA polymerase: insights into the mechanisms of viral RNA synthesis. Nat. Rev. Microbiol. 14, 479-493 (2016).

46 Byrn, R. A. et al. Preclinical activity of VX-787, a first-in-class, orally bioavailable inhibitor of the influenza virus polymerase PB2 subunit. Antimicrob. Agents Chemother. 59, 1569-1582 (2015).

47 Clark, M. P. et al. Discovery of a novel, first-in-class, orally bioavailable azaindole inhibitor (VX-787) of influenza PB2. J. Med. Chem. 57, 6668-6678 (2014).

48 Sidwell, R. W. et al. Broad-spectrum antiviral activity of virazole: 1-f8- D-ribofuranosyl1,2,4-triazole- 3-carboxamide. Science 177, 705-706 (1972).

49 Eriksson, B. et al. Inhibition of influenza virus ribonucleic acid polymerase by ribavirin triphosphate inhibition of influenza virus ribonucleic acid polymerase by ribavirin triphosphate. Antimicrob. Agents Chemother. 11, 946-951 (1977).

50 Furuta, Y. et al. In vitro and in vivo activities of anti-influenza virus compound T-705. Antimicrob. Agents Chemother. 46, 977-981 (2002).

51 Furuta, Y. et al. Mechanism of action of T-705 against influenza virus. Antimicrob. Agents Chemother. 49, 981-986 (2005).

52 Jin, Z., Smith, L. K., Rajwanshi, V. K., Kim, B. \& Deval, J. The ambiguous basepairing and high substrate efficiency of T-705 (Favipiravir) Ribofuranosyl 5'-triphosphate towards influenza A virus polymerase. PLOS ONE 8, e68347 (2013).

53 Baranovich, T. et al. T-705 (Favipiravir) induces lethal mutagenesis in influenza A H1N1 viruses in vitro. J. Virol. 87, 3741-3751 (2013).

54 Sangawa, $\mathrm{H}$. et al. Mechanism of action of T-705 ribosyl triphosphate against influenza virus RNA polymerase. Antimicrob. Agents Chemother. 57, 5202-5208 (2013).

55 Grienke, U. et al. Influenza neuraminidase: a druggable target for natural products. Nat. Prod. Rep. 29, 11-36 (2012).

56 Minagawa, K., Kouzuki, S. \& Kamigauchi, T. Stachyflin and acetylstachyflin, nove anti-influenza A virus substances, produced by Stachybotrys sp. RF-7260. II. Synthesis and preliminary structure-activity relationships of stachyflin derivatives. $J$. Antibiot. (Tokyo) 55, 165-171 (2002).

57 Minagawa, K. et al. Stachyflin and acetylstachyflin, novel anti-influenza A virus substances, produced by Stachybotrys sp. RF-7260. I. Isolation, structure elucidation and biological activities. J. Antibiot. (Tokyo) 55, 155-164 (2002).

58 Nakatani, M., Nakamura, M., Suzuki, A., Inoue, M. \& Katoh, T. A new strategy toward the total synthesis of stachyflin, a potent anti-influenza a virus agent: concise route to the tetracyclic core structure. Org. Lett. 4, 4483-4486 (2002).

59 World Health Organization. Global Health Sector Strategy on Viral Hepatitis 20162021 (World Health Organization, Geneva, Switzerland, 2016).

60 World Health Organization Media centre. Fact sheets. Hepatitis B (2017) http://www. who.int/mediacentre/factsheets/fs204/en/ Accessed 27 May 2017.

61 Zhang, F. \& Wang, G. A review of non-nucleoside anti-hepatitis B virus agents. Eur. J. Med. Chem. 75, 267-281 (2014).

$62 \mathrm{Wu}, \mathrm{Y} .-\mathrm{H}$. Naturally derived anti-hepatitis B virus agents and their mechanism of action. World J. Gastroenterol. 22, 188-204 (2016).

63 Yan, H. et al. Sodium taurocholate cotransporting polypeptide is a functional receptor for human hepatitis B and D virus. Elife 1, 1-28 (2012).

64 Hepatitis B Foundation. Treatment \& Management. Drug Watch (2017) http://www. hepb.org/treatment-and-management/drug-watch/ Accessed 27 May 2017.

65 Manzoor, S. Hepatitis B virus therapy: what's the future holding for us? World J. Gastroenterol. 21, 12558 (2015).

66 Nagahata, T., Kitagawa, M. \& Matsubara, K. Effect of oxetanocin G, a novel nucleoside analog, on DNA synthesis by hepatitis B virus virions. Antimicrob. Agents Chemother. 38, 707-712 (1994). 
67 Nagahata, T., Ueda, K., Tsurimoto, T., Chisaka, O. \& Matsubara, K. Anti-hepatitis B virus activities of purine derivatives of oxetanocin A. J. Antibiot. (Tokyo) 42, 644-646 (1989).

68 Wang, X. Emerging antivirals for the treatment of hepatitis B. World J. Gastroenterol. 20, 7707 (2014).

69 Summers, J., Smith, P. M. \& Horwich, A. L. Hepadnavirus envelope proteins regulate covalently closed circular DNA amplification. J. Virol. 64, 2819-2824 (1990).

70 Levrero, M. et al. Control of cccDNA function in hepatitis B virus infection. J. Hepatol. 51, 581-592 (2009).

71 Yang, L. et al. Isothiafludine, a novel non-nucleoside compound, inhibits hepatitis B virus replication through blocking pregenomic RNA encapsidation. Acta Pharmacol. Sin. 35, 410-418 (2014).

72 Yang, L. et al. Effect of a hepatitis B virus inhibitor, NZ-4, on capsid formation. Antiviral Res. 125, 25-33 (2016).

$73 \mathrm{Hu}$, Y., Cheng, X., Cao, F., Huang, A. \& Tavis, J. E. $\beta$-Thujaplicinol inhibits hepatitis B virus replication by blocking the viral ribonuclease $\mathrm{H}$ activity. Antiviral Res. 99, 221-229 (2013).

$74 \mathrm{Lu}, \mathrm{G}$. et al. Hydroxylated tropolones inhibit hepatitis B virus replication by blocking viral ribonuclease H activity. Antimicrob. Agents Chemother. 59, 1070-1079 (2015).

75 Kitamura, S., lida, T. Shirahata, K \& Kase, H. Studies on lipoxygenase inhibitors. I. MY3-469 (3-methoxytropolone), a potent and selective inhibitor of 12-lipoxygenase, produced by Streptoverticillium hadanonense KY11449. J. Antibiot. (Tokyo) 39, 589-593 (1986).

76 Suzuki, M. et al. Efficient genome replication of hepatitis B virus using adenovirus vector: a compact pregenomic RNA-expression unit. Sci. Rep. 7, 41851 (2017).

77 Gehring, A., Bertoletti, A. \& Tavis, J. E. Host factor-targeted hepatitis B virus therapies. Intervirology 57, 158-162 (2014).

78 Ezzikouri, S. et al. Recent insights into hepatitis B virus-host interactions. J. Med. Virol. 86, 925-932 (2014).

79 Gripon, P., Cannie, I. \& Urban, S. Efficient inhibition of hepatitis B virus infection by acylated peptides derived from the large viral surface protein. J. Virol. 79, 1613-1622 (2005).

80 Volz, T. et al. The entry inhibitor Myrcludex-B efficiently blocks intrahepatic virus spreading in humanized mice previously infected with hepatitis B virus. J. Hepatol. 58, 861-867 (2013).

81 Nkongolo, S. et al. Cyclosporin A inhibits hepatitis B and hepatitis D virus entry by cyclophilin-independent interference with the NTCP receptor. J. Hepatol. 60, 723-731 (2014).

82 Watashi, K. et al. Cyclosporin A and its analogs inhibit hepatitis B virus entry into cultured hepatocytes through targeting a membrane transporter, sodium taurocholate cotransporting polypeptide (NTCP). Hepatology 59, 1726-1737 (2014).

83 Block, T. M. et al. Secretion of human hepatitis B virus is inhibited by the imino sugar N-butyldeoxynojirimycin. Proc. Natl Acad. Sci. USA 91, 2235-2239 (1994).

84 Block, T. M. et al. Treatment of chronic hepadnavirus infection in a woodchuck animal model with an inhibitor of protein folding and trafficking. Nat. Med. 4, 610-614 (1998).

85 Alonzi, D. S., Scott, K. A., Dwek, R. A. \& Zitzmann, N. Iminosugar antivirals: the therapeutic sweet spot. Biochem. Soc. Trans. 45, 571-582 (2017).

86 Umezawa, H., Aoyagi, T., Suda, H., Hamada, M. \& Takeuchi, T. Bestatin, an inhibitor of aminopeptidase B, produced by actinomycetes. J. Antibiot. (Tokyo) 29, 97-99 (1976).

87 Sasaki, S. et al. Adjuvant effect of Ubenimex on a DNA vaccine for HIV-1. Clin. Exp. Immunol. 111, 30-35 (1998).

88 Wright, P. J., Crameri, G. \& Eaton, B. T. RNA synthesis during infection by Hendra virus: an examination by quantitative real-time PCR of RNA accumulation, the effect of ribavirin and the attenuation of transcription. Arch. Virol. 150, 521-532 (2005).

89 Chang, K.-O. \& George, D. W. Interferons and Ribavirin effectively inhibit norwalk virus replication in replicon-bearing cells. J. Virol. 81, 12111-12118 (2007).

90 Koff, W. C., Elm, J. L. \& Halstead, S. B. Antiviral effects if ribavirin and 6-mercapto-9tetrahydro-2-furylpurine against dengue viruses in vitro. Antiviral Res. 2, 69-79 (1982).

91 Streeter, D. G. et al. Mechanism of action of 1- -D-ribofuranosyl-1,2,4-triazole-3carboxamide (Virazole), a new broad-spectrum antiviral agent. Proc. Natl Acad. Sci. USA 70, 1174-1178 (1973).

92 Oestereich, L. et al. Successful treatment of advanced Ebola virus infection with T-705 (favipiravir) in a small animal model. Antiviral Res. 105, 17-21 (2014).

93 Smither, S. J. et al. Post-exposure efficacy of Oral T-705 (Favipiravir) against inhalational Ebola virus infection in a mouse model. Antiviral Res 104, 153-155 (2014).
94 Warren, T. K. et al. Protection against filovirus diseases by a novel broad-spectrum nucleoside analogue BCX4430. Nature 508, 402-405 (2014).

95 Warren, T. K. et al. Therapeutic efficacy of the small molecule GS-5734 against Ebola virus in rhesus monkeys. Nature 531, 381-385 (2016).

96 Eyer, L. et al. Antiviral activity of the adenosine analogue BCX4430 against West Nile virus and tick-borne flaviviruses. Antiviral Res. 142, 63-67 (2017).

97 Julander, J. G. et al. BCX4430, a novel nucleoside analog, effectively treats yellow fever in a hamster model. Antimicrob. Agents Chemother. 58, 6607-6614 (2014).

98 Julander, J. G. et al. Efficacy of the broad-spectrum antiviral compound BCX4430 against Zika virus in cell culture and in a mouse model. Antiviral Res. 137, 14-22 (2017).

99 Lo, M. K. et al. GS-5734 and its parent nucleoside analog inhibit Filo-, Pneumo-, and Paramyxoviruses. Sci. Rep. 7, 43395 (2017).

100 Endo, A., Kuroda, M. \& Tanzawa, K. Competitive inhibition of 3-hydroxy-3methylglutaryl coenzyme a reductase by ML-236A and ML-236B fungal metabolites, having hypocholesterolemic activity. FEBS Lett. 72, 323-326 (1976).

101 Martínez-Gutierrez, M., Castellanos, J. E. \& Gallego-Gómez, J. C. Statins reduce dengue virus production via decreased virion assembly. Intervirology 54, 202-216 (2011).

$102 \mathrm{Ikeda}, \mathrm{M}$. et al. Different anti-HCV profiles of statins and their potential for combination therapy with interferon. Hepatology 44, 117-125 (2006).

103 Peng, J. et al. Protective effect of fluvastatin on influenza virus infection. Mol. Med. Rep. 9, 2221-2226 (2014).

104 Giguère, J. \& Tremblay, M. J. Statin compounds reduce human immunodeficiency virus type 1 replication by preventing the interaction between virion-associated host intercellular adhesion molecule 1 and its natural cell statin compounds reduce human immunodeficiency virus type 1 replica. J. Virol. 78, 1-5 (2004).

105 Bader, T. \& Korba, B. Simvastatin potentiates the anti-hepatitis B virus activity of FDA-approved nucleoside analogue inhibitors in vitro. Antiviral Res. 86, 241-245 (2010).

106 Sadanari, H. et al. Inhibitory effects of statins on expression of immediate-early 1 protein of human cytomegalovirus in virus-infected cells. J. Exp. Clin. Med. 5, 187-193 (2013).

107 Kluepfel, D. et al. Myoriocin, a new antifungal antibiotic from Myriococcum arbomyces. J. Antibiot. (Tokyo) 22, 109-115 (1972).

108 Sakamoto, H. et al. Host sphingolipid biosynthesis as a target for hepatitis C virus therapy. Nat. Chem. Biol. 1, 333-337 (2005).

109 Tatematsu, K., Tanaka, Y., Sugiyama, M., Sudoh, M. \& Mizokami, M. Host sphingolipid biosynthesis is a promising therapeutic target for the inhibition of hepatitis B virus replication. J. Med. Virol. 83, 587-593 (2011).

110 Tafesse, F. G. et al. Intact sphingomyelin biosynthetic pathway is essential for intracellular transport of influenza virus glycoproteins. Proc. Natl Acad. Sci. USA 110, 6406-6411 (2013).

111 Bienkowska-Haba, M., Williams, C., Kim, S. M., Garcea, R. L. \& Sapp, M. Cyclophilins facilitate dissociation of the human papillomavirus type 16 capsid protein $L 1$ from the L2/DNA complex following virus entry. J. Virol. 86, 9875-9887 (2012).

112 Karpas, A., Lowdell, M., Jacobson, S. K. \& Hill, F. Inhibition of human immunodeficiency virus and growth of infected $T$ cells by the immunosuppressive drugs cyclosporin A and FK 506. Proc. Natl Acad. Sci. USA 89, 8351-8355 (1992).

113 Pfefferle, S. et al. The SARS-coronavirus-host interactome: identification of cyclophilins as target for pan-coronavirus inhibitors. PLoS Pathog. 7, e1002331 (2011).

114 Liu, X. et al. Cyclosporin A inhibits the influenza virus replication through cyclophilin A-dependent and -independent pathways. PLOS ONE 7, 1-8 (2012).

115 Keyes, L. R., Bego, M. G., Soland, M. \& St. Jeor, S. Cyclophilin A is required for efficient human cytomegalovirus DNA replication and reactivation. J. Gen. Virol. 93, 722-732 (2012).

116 Watashi, K., Hijikata, M., Hosaka, M., Yamaji, M. \& Shimotohno, K. Cyclosporin A suppresses replication of hepatitis $C$ virus genome in cultured hepatocytes. Hepatology 38, 1282-1288 (2003).

117 Matsuda, S. \& Koyasu, S. Mechanisms of action of cyclosporine. Immunopharmacology 47, 119-125 (2000).

118 Peel, M. \& Scribner, A. Cyclophilin inhibitors as antiviral agents. Bioorganic Med. Chem. Lett. 23, 4485-4492 (2013).

119 Chatterji, U. et al. The combination of alisporivir plus an NS5A inhibitor provides additive to synergistic anti-hepatitis $\mathrm{c}$ virus activity without detectable crossresistance. Antimicrob. Agents Chemother. 58, 3327-3334 (2014). 